\title{
LA FEMINIZACIÓN DE LA SUPERVIVENCIA EN CONTEXTOS MIGRATORIOS Y MULTICULTURALES
}

\author{
Gloria Patricia Lopera Mesa \\ Yulieth Teresa Hillón Vega
}

\author{
Escuela de Derecho de la Universidad EAFIT \\ Medellín (Colombia)
}

\begin{abstract}
Resumen
Este documento hace una aproximación preliminar a las interrelaciones entre las injusticias de distribución y de reconocimiento presentes en las desigualdades de género, centrándose en dos poblaciones que pueden ser consideradas como minorías culturales. El escrito está dividido en tres partes. En un primer momento, definiremos los parámetros teóricos desde los cuáles partió esta exploración. En segundo lugar, presentaremos dos casos en los cuales se evidencia esa interrelación de las estructuras sociales, económicas, culturales y de género. Por una parte, hablaremos de algunas de las desigualdades a las que se enfrentan las migrantes colombianas en su viaje a España y la forma como construyen la masculinidad y feminidad en ese país. Por otra, estudiaremos la relación entre esas injusticias a partir del modo en que los medios de comunicación presentaron el caso de las indígenas Embera del Chocó (Colombia) que son sancionadas por su comunidad por cortar su cabello para venderlo en las peluquerías de Quibdó, capital de ese departamento. Finalizaremos con algunas conclusiones que, más que respuestas, son perspectivas de análisis que podrían orientar futuras investigaciones.
\end{abstract}

- Palabras clave: Relaciones familiares y grupales; Desigualdad de género; Reconocimiento legal; Grupos étnicos; Poblaciones migrantes

Abstract

- Keywords: Family relationships; Ties into cultural groups; Gender inequity; Legal recognition; Ethnics groups; Migrant people
This paper raises a rapproachment to unjust economic distribution and continue failure to recognise women rights that is linked to gender disparity. Authors focusing on two sectors of Colombian people: Ethnics groups who live in the interior of the country, more precisely, the Embera - Chocó, and migrant women who travel to Spain looking for improve their wellfare. 
I. Las injusticias de reconocimiento y de distribución dentro de las desigualdades de género - II. Las mujeres colombianas migrantes en España - III. El cubrimiento en los medios de la sanción impuesta a las mujeres Embera por vender su cabello - IV. Conclusiones

\section{LAS INJUSTICIAS DE RECONOCIMIENTO Y DE DISTRIBUCIÓN DENTRO DE LAS DESIGUALDADES DE GÉNERO}

Antes de entrar en el análisis de los casos, es preciso presentar los puntos de partida que servirán como marco de análisis.

En primer lugar, vamos a retomar algunos elementos de la teoría de la justicia propuesta por Nancy Fraser. Esta autora señala que una sociedad que avanza hacia la justicia social (hacia una "democracia radical") es aquella que se propone erradicar dos tipos de injusticias: (a) las que se derivan de la inequitativa distribución de la riqueza y (b) las que se originan en la falta de reconocimiento y respeto por las diversas identidades, esto es, por las distintas formas que tenemos las personas de dotar de sentido y vivenciar nuestra común humanidad, debido a la construcción de patrones culturales que devalúan o menosprecian ciertas formas de ser humano ${ }^{1}$.

La virtud que tiene la aproximación que propone Fraser es que logra superar la tendencia a equiparar política de redistribución con solución a las injusticias de clase y política de la identidad como solución a las injusticias de género, sexualidad y raza. En su lugar, prefiere tratar la redistribución y el reconocimiento como dimensiones de la justicia que atraviesan las demandas formuladas por todos los movimientos sociales.

Es raro encontrar situaciones de injusticia que tengan su origen sólo en problemas de redistribución o reconocimiento. Cuando examinamos las situaciones de injusticia padecidas por un grupo social, vemos que estas dos variantes suelen aparecer mezcladas y retroalimentarse mutuamente. Así, señala esta autora, las discriminaciones por razón de género aúnan tanto injusticias de redistribución (por el distinto papel que ocupan mujeres y hombres en la estructura productiva de la sociedad) como de reconocimiento (por el androcentrismo cultural). Lo mismo podría afirmarse cuando analizamos las situaciones de injusticia presentes en fenómenos sociales como las migraciones o las que padecen las minorías étnicas. En los dos casos que examinaremos a continuación, trataremos de analizar de qué manera se manifiestan y se relacionan ambas fuentes de injusticia en contra de las mujeres migrantes y de las mujeres indígenas.

En segundo lugar, si hemos anunciado que el objetivo central de esta ponencia es formular preguntas que incorporen una mirada de género a los temas que actualmente constituyen nuestros temas de reflexión, es pertinente aclarar en qué sentido emplearemos las nociones de "género" y “patriarcado”; ligado a ello, en qué

${ }^{1}$ FRASER, N., Iustitia Interrupta. Reflexiones críticas desde la posición "postsocialista”, trad. M. Holguín e I. C. Jaramillo, Bogotá, Siglo del Hombre, 1997, pp. 20 y ss. 
sentido hablamos de "feminización de la pobreza" y de su corolario, la "feminización de la supervivencia". En este punto nos valdremos de la aproximación que propone la antropóloga argentina, radicada en Brasil, Rita Laura Segato ${ }^{2}$.

Según explica esta autora, el género constituye un principio de clasificación y de construcción de alteridad, basado en la distinción entre lo "masculino" y lo "femenino" , la cual suele equipararse, aun cuando no equivale, a la distinción biológica entre "hombre" y "mujer".

La fuerza de este principio clasificatorio básico parecía descansar, y por mucho tiempo así se pensó, en diferencias biológicas entre hombre y mujer. Desde esta perspectiva, la diferencia biológica era el dato previo que respaldaba y naturalizaba las diferencias de género. Es la biología la que explica y justifica que los hombres salgan a cazar (y en general a proveer), realicen las labores que demandan mayor capacidad física e intelectual, y que las mujeres se ocupen de procrear y cuidar de su progenie. Sobre tales diferencias biológicas se hacía descansar cierta concepción acerca de los roles masculino y femenino. Sin embargo, la primera generación de estudios en antropología de género, encabezada por los trabajos de Margaret Mead, mostró que la distinción entre masculino y femenino y, más aún, de la discontinuidad biológica entre hombre y mujer, son construcciones culturales, lo que contribuyó a cimentar la crítica al sexismo, esto es, a las formas de discriminación que se justifican apelando a diferencias biológico sexuales que determinarían las facultades y comportamientos distintos que asumen (y deben asumir) hombres y mujeres.

Con todo, los trabajos de la antropología de género también permitieron establecer que en las diversas sociedades lo masculino se construye como una identidad que se afirma a partir de la dominación, del control, sobre lo femenino. Con esto no se quiere indicar sólo que quienes ocupan una posición masculina en la relación social afirmen su masculinidad a partir de la dominación y del control sobre ese "otro" que en esa relación social ocupe el rol femenino. También, visto desde la perspectiva del propio individuo, su masculinidad se afirma a partir de negar, controlar, suprimir aquellos rasgos de su ser que delatan la presencia de lo femenino.

Rita Segato sostiene que el género no puede caracterizarse como un conjunto de comportamientos documentables que consiste en lo que los hombres y las mujeres hacen en una determinada sociedad, sino más bien como una estructura de relaciones entre sujetos que ocupan el lugar de lo 'femenino' y de lo 'masculino', pero que no necesariamente coinciden con las anatomías de mujer / hombre. En ese orden de ideas, se pregunta esta autora, "Si los géneros fuesen simplemente lo que los hombres y las mujeres hacen de forma diferenciada, ¿cómo podríamos explicar, por ejemplo, el resurgimiento de la estructura de género en el interior de las instituciones totales como cárceles y conventos, pero escenificada allí por actores de anatomías idénticas?”3.

2 SEGATO, R. L., Las estructuras elementales de la violencia. Ensayos sobre género entre la antropología, el psicoanálisis y los derechos humanos, Bernal, Universidad Nacional de Quilmes, 2003.

3 Ibidem, p. 74. 
Se emplea la noción de "patriarcado" para nombrar a dicha estructura jerárquica de relaciones sociales basada en la construcción de una identidad masculina que se edifica y reafirma a partir del ejercicio de dominación sobre lo femenino. El patriarcado constituye una de las manifestaciones de la dialéctica del amo y del esclavo de la que hablara Hegel, en virtud de la cual el amo solo puede existir como tal si a su vez existe un otro, en posición de esclavo, sobre cuya subordinación pueda el amo construir su identidad de amo. Se trata de una estructura de dominación que atraviesa fronteras culturales, y que no es exclusiva de las relaciones de género, sino de todas las identidades que se definen y construyen a partir del ejercicio de poder y superioridad respecto de otros. También está en la base de la estructura de dominación colonial respecto de los pueblos colonizados, del discurso que afirma la superioridad de las gentes blancas sobre las no-blancas. De ahí que los estudios de género, enfocados en develar las múltiples manifestaciones de la dominación patriarcal, hayan abierto el camino y estén directamente relacionados con los estudios sobre subalternidad y minorías sociales.

Para Segato, en las relaciones basadas en el estatus, la plenitud del ser de quienes ocupan un rol dominante (masculino, blanco, imperio) depende de un ser-menos de los que participan como "otros” dentro del sistema. Este ser-menos o minusvalía sólo puede ser el resultado de una exacción o expropiación simbólica y material que reduce la plenitud de estos últimos a fin de alimentar la de aquellos ${ }^{4}$.

Pero ¿cómo se sostiene la dominación de género? El cimiento sobre el cual se constituye y reproduce la dominación patriarcal son las múltiples formas de lo que Rita Laura Segato define como "violencia moral", concepto que emplea para nombrar todas las formas sutiles de discriminación y menosprecio hacia la mujer y, en general, hacia quienes ocupan una posición minorizada en un sistema de estatus. Formas de violencia que incluyen el control económico, de la sociabilidad, de la movilidad, de los cuerpos, menosprecio moral, estético, sexual, descalificación intelectual o profesional ${ }^{5}$.

Estas diversas formas de violencia aúnan lo que Fraser denominaría injusticias tanto de redistribución, derivadas del desigual reparto de bienes y acceso a oportunidades entre quienes ocupan roles masculinos y femeninos, como de reconocimiento, originadas en la preferencia por los atributos asociados a lo masculino respecto de los que se atribuyen a lo femenino, y que se ejemplifica en un sinnúmero de prácticas y expresiones tales como la predilección por engendrar niños en lugar de niñas, que en países como India y China ha llevado al infanticidio masivo de niñas ${ }^{6}$; en la utilización entre nuestros jóvenes del adjetivo "eso es muy gay" para descalificar el uso de ciertas prendas de vestir o de ciertos comportamientos que pueden delatar la presencia de lo femenino, e inclusive en la misoginia que se evidencia en la utilización en el mundo gay masculino de términos como "loca” o

\footnotetext{
${ }^{4}$ Ibidem, p. 254.

${ }^{5}$ Ibidem, p. 116.

${ }^{6}$ PERROT, M., Mi historia de las mujeres, trad. M. Saúl, Buenos Aires, Fondo de Cultura Económica, 2009, p. 53.
} 
“mostrar las plumas” para descalificar y situar por debajo del "macho” a todo aquél, hombre o mujer, que se aproxime a lo femenino".

Algo interesante del trabajo de Rita Segato es que muestra cómo las diversas violencias que constituyen la dominación patriarcal no afectan sólo a quienes ocupan el rol femenino (que en la mayoría de ocasiones son las mujeres), sino a los propios individuos que, impelidos por el mandato de afirmar su masculinidad, construyen su identidad masculina a partir de la represión de lo femenino que habita en ellos. En tal sentido, señala esta autora que "el sexismo debe ser reconocido como un problema de los hombres, cuya humanidad se deteriora y se degrada al ser presionados por la moral tradicional y por el régimen de estatus a reconducirse todos los días, por la fuerza o por la maña, a su posición de dominación”8.

A propósito de esto, conviene abrir un paréntesis en el hilo de nuestro discurso para revisar críticamente esa curiosa división del trabajo intelectual que se ha impuesto, según la cual la reflexión sobre género, al igual que antaño ocurría con la cocina, parecería ser un espacio a ocupar sólo por mujeres, que interesa sólo a mujeres y en el que aquel hombre que se atreva a incursionar se arriesga a la mirada sospechosa de sus congéneres. División del trabajo que se pone de manifiesto en que hayamos sido nosotras, en lugar de otros de nuestros compañeros hombres de la Escuela de Derecho, las invitadas a presentar hoy esta reflexión ante ustedes. Dado que pensar el género es pensar en la relación entre masculino y femenino, es algo que atañe por igual a hombres y mujeres, pues todos nos relacionamos con nosotros mismos y con los demás a partir de un esquema de constitución de la alteridad basado en la distinción entre lo masculino y lo femenino. Y si se argumenta que la pregunta por el género es algo que reviste mayor interés para las mujeres, pues son éstas quienes en su mayoría padecen los efectos de las diversas formas de violencia que alimentan la dominación patriarcal, habría que contestar que esta violencia, de manera directa e indirecta, también afecta a quienes se ven forzados a construir su identidad masculina a partir de una permanente negación y dominación de lo femenino.

Cerrado este paréntesis, uno de los resultados visibles del cúmulo de injusticias de redistribución y de reconocimiento a través de las cuales se expresa y reproduce el patriarcado, es la denominada "feminización de la pobreza". Se trata de un concepto empleado en los estudios de género y en la Plataforma de Acción suscrita en la Conferencia de Beijing de 1995 para nombrar el inequitativo reparto de la riqueza que discrimina a las mujeres, y que se manifiesta de modo elocuente en el hecho de que la mayoría de la población pobre en el mundo, esto es, de los 1500 millones de personas que vive con menos de un dólar al día, está conformada por mujeres. También en los estudios que demuestran que, en todo el mundo, las mujeres ganan en promedio un $50 \%$ menos de lo que ganan los hombres.

Como señalan Rosa Cobo y Luisa Posada en la presentación a su estudio sobre el tema, aun cuando no pueden negarse los cambios experimentados en la posición

${ }^{7}$ PONSFORD, M., “¿Sin una pluma?”, Editorial de Arcadia, No. 69, 20 de junio al 15 de julio de 2011.

8 SEGATO, R. L., op. cit., p. 146. 
social de las mujeres derivados de su acceso al mercado de trabajo y de la conquista de derechos políticos, lo cierto es que, a la par, uno de los efectos más rotundos de los programas de ajuste estructural inherentes a las políticas neoliberales es el crecimiento del trabajo gratuito de las mujeres en el hogar, resultado de los recortes de los programas sociales por parte de los gobiernos. Aquellas funciones de las que el Estado abdica (o que nunca asumió por completo, añado) -como la garantía de la salud o nutrición o educación, entre otras- vuelven a recaer en la familia, específicamente en las mujeres, lo que les impide acceder a trabajos que requieran dedicación exclusiva ${ }^{9}$.

Esta situación ha llevado también a lo que Saskia Sassen denomina "feminización de la supervivencia”10 . Si partimos del hecho de que las mujeres son en mayor medida víctimas de la pobreza y que sobre ellas recaen las tareas de reproducción y cuidado familiar, llegando muchas veces a ser las cabezas de familia, se comprende la necesidad de que éstas se lancen a buscar alternativas para garantizar no sólo su propia supervivencia, sino la de aquellos que están bajo su cuidado. De ahí que la producción alimenticia de subsistencia, el trabajo informal, la prostitución y la emigración, entre otras, se convierten en los caminos que emprenden las mujeres en su lucha por subsistir, por sacar a los seres queridos adelante (y cada vez más a las economías de sus países de origen). Precisamente los dos casos que presentamos a continuación constituyen una muestra de estas estrategias de "feminización de la supervivencia”.

\section{LAS MUJERES COLOMBIANAS MIGRANTES EN ESPAÑA ${ }^{11}$}

Variados son los aspectos que evidencian las interacciones entre el género, la economía, la cultura o la violencia y sus desigualdades en el caso de las mujeres migrantes colombianas en España. Sin embargo, centraremos nuestra exposición en dos elementos que muestran de forma fehaciente esas conexiones mezcladas y metamorfoseadas. Por un lado, constataremos cómo el gran peso que las mujeres tienen dentro del flujo colombiano hacia España y los motivos que arguyen para dejar su país reflejan una serie de inequidades de género, conectadas con injusticias de diversos tipos, que las llevan a buscar la migración como vía de escape y resistencia. Por otro, teniendo en cuenta que uno de los elementos que utilizan los colombianos en España para forjarse una identidad étnica que los diferencie de los españoles es la distinta manera de construir la relación entre hombre y mujer, veremos que existe una autopercepción idealizada de las relaciones de pareja que

9 COBO, R, y POSADA, L., La feminización de la pobreza, El País, 15/06/2006, http://www.elpais.com. Última revisión, Julio 1/2011.

10 SASSEN, S., Contrageografías de la globalización. Género y ciudadanía en los circuitos transfronterizos, Madrid, 2003.

11 Las reflexiones consignadas en este acápite parten del material cualitativo recogido para la tesis doctoral La estrategia del caracol. Migración y transformación social. El caso de los colombianos en Aragón. Para mayor información al respecto, ver HILLÓN, Y., El caracol en su laberinto. Migración y transformación social. El caso de los colombianos en Aragón, Oñati, 2011 (En proceso de publicación). 
refuerza y retroalimenta concepciones de feminidad y masculinidad propias de un sistema patriarcal compartido tanto en la sociedad de origen como en la de llegada.

Comenzando con el primer punto, la participación de la mujer en los flujos migratorios globales ha dejado huella. Incluso antes de los años setenta del siglo pasado, década en la que surgió una nueva etapa en los movimientos humanos (las migraciones contemporáneas) y que suele caracterizarse por la feminización del fenómeno $^{12}$, alrededor del $50 \%$ de los migrantes ya eran mujeres ${ }^{13}$. Colombia no es la excepción. En 2008, el 51.4\% de los colombianos viviendo en el extranjero eran féminas ${ }^{14}$.

Esa huella femenina caracteriza de forma más marcada el flujo migratorio colombiano hacia España, si bien su fuerte presencia ha menguado con el tiempo. En 2001, año en el que la migración de colombianos hacia la Península comenzó a tomar vuelo, el 60\% eran mujeres; para inicios de 2010, esta cifra se había reducido a un no despreciable $55,2 \%{ }^{15}$. Esta tendencia no debe sorprendernos: al ser ellas las pioneras del movimiento hacia la Ibérica, son las que con su esfuerzo y la ayuda de la demanda de mano de obra para la construcción y el sector servicios han traído a sus familiares y amigos. Es decir, más mujeres de su círculo, por lo que continúa el patrón, pero también compañeros sentimentales, hijos, hermanos, familiares o amigos, lo que explica la reducción del porcentaje. Adelaida, una de las participantes en el estudio cualitativo, nos explica la dinámica:

"Carolina, ponle tres primas aquí. Llega Carolina, Esteban... voy a hablarte por mi lado, ¿vale? Esteban, Inés y Enrique, Matías, Luis y Margarita, Lucia, Rebeca y Adelaida, Úrsula, Aureliano, Mateo... Van 15 personas por el lado nuestro. Por el lado de ella (Carolina) y posterior a ella están: Ángela, Susana, David (...) Nubia, Melisa, la niña que acaban de traer, que no sé cómo se llama. Van 20 personas. Un primo de ellos... por lo menos cuatro personas más. 20 personas. Que es una cadena. Y como ésta, muchas. Y eso que no te cuento los de Catalina.... Como ésta, muchísimas, muchísimas, muchísimas. Y yo creo que el proceso, por el lado nuestro, se viene a terminar con la venida de Valeria... de

$12 \mathrm{Al}$ respecto, ver, entre otros, BADE, K., Europa en movimiento. Las migraciones desde finales del siglo XVIII hasta nuestros días, Barcelona, 2003; BLANCO, C., Las migraciones contemporáneas, Madrid, 2000; CASTLES, S., y MILLER M., The Age of Migration. International Population Movements in the Modern World, Houndmills, Basingstoke, Hampshire and London, 1993; o STOLCKE, V, Qué entendemos por integración social de los inmigrantes, en CHECA OLMOS, F, CHECA, J. C. y ARJONA, A., Inmigración y derechos humanos. La integración como participación social, Barcelona, 2004, pp. 17 - 45.

13 OIM, World Migration 2005: Costs and Benefits of International Migration, Geneva, 2005; UNDESA, International Migration 2006: A Global Assessment, New York, 2009.

14 DAS, DANE, OIM, Anuario Estadístico, Entradas y Salidas Internacionales, Colombia 2006-2007, Bogotá, 2008.

15 INE, Revisión del Padrón Municipal 2002. Datos a nivel nacional, comunidad autónoma y provincia, España, 2002; INE, Revisión del Padrón Municipal 2010. Datos a nivel nacional, comunidad autónoma y provincia, España, 2011. 
mi prima. Pero Valeria, a su vez, se traerá más tarde o más temprano a su hija, se traerá a su madre y a su hermana. Y tras de ellos, vendrán más”.

Ahora bien, ¿Por qué salieron esos colombianos y sobre todo esas colombianas del país? Fuerzas internas y externas, muchas de ellas bastante conocidas, se confabularon para que esto tuviera lugar. Según la definición de "inmigrante" utilizada por los medios académicos, legislativos y mediáticos y por la comunidad en general, incluidos los mismos implicados -ciudadanos pobres del tercer mundo en el primer mundo-, la raíz de su viaje se encuentra en su faceta económica. Este hecho se torna más evidente si recordamos las situaciones colombiana y española en ese aspecto para la época en que creció el flujo migratorio colombiano ${ }^{16}$. En España, había un despegue económico formal e informal que clamaba a gritos por mano de obra; específicamente, personal para el trabajo doméstico, el cuidado de niños y ancianos, la construcción y la hostelería. Mientras tanto, Colombia pasaba por una grave situación económica y de orden público a finales de los 90 y principios de este siglo, la cual se veía agravada por una serie de catástrofes naturales, como por ejemplo, el terremoto de Armenia (Quindío) en el Eje Cafetero ${ }^{17}$. Dada esta difícil coyuntura, las familias colombianas encontraron una salida a esa situación migrando hacia la Ibérica, lugar en el que en ese momento se necesitaba trabajadores y no se requería visa para viajar ${ }^{18}$.

Sin embargo, el factor económico no explica completamente la migración en general y la migración colombiana en particular. Al escudriñar las historias de las personas entrevistadas, la motivación económica se encuentra presente; no obstante, en sus relatos se mezclan una gran variedad de razones. Tenemos cuestiones afectivas, académicas, políticas, económicas, culturales... En el caso de las mujeres, muchos de esos motivos están conectados con desigualdades de género que se reflejan en diferentes injusticias, algunas veces, encubiertas. Unos cuantos ejemplos nos permitirán ilustrar claramente estas inequidades de las que estamos hablando.

16 Como ya se había anotado, el nuevo milenio marcó el auge de la migración de colombianos hacia España. Hasta el año 2000, los 24.702 nacionales de ese territorio latinoamericano que residían en la Ibérica se sumergían dentro de la categoría "resto de nacionalidades de América del Sur”, aunque su incremento era evidente. Dos años después, el panorama era completamente diferente. Para 2002, las cifras se dispararon a 191.018. Al respecto, ver INE, Revisión del Padrón Municipal 2000. Datos a nivel nacional, comunidad autónoma y provincia, España, 2000; INE, Revisión del Padrón Municipal 2002. Datos a nivel nacional, comunidad autónoma y provincia, España, 2002.

17 El 25 de enero de 1999 un fuerte terremoto tuvo lugar en la zona cafetera colombiana, afectando especialmente al departamento del Quindío (Armenia y zonas aledañas). Además de las vidas que cobró, el desastre natural arrasó con gran parte de la infraestructura regional urbana y rural, generando un impacto social, económico y laboral sin precedentes.

18 Es importante advertir que las continuas amenazas sobre la imposición de "la visa" por parte de las autoridades españolas y su efectiva puesta en marcha el 2 de enero de 2002 crearon una especie de alarma entre aquellos deseosos por viajar e incentivaron la venida rápida y apresurada de miles de personas antes de que "las puertas se cerraran”. 
Dentro de las motivaciones de salida encontramos la violencia endémica que vive el país. El 43\% de las solicitudes de asilo en España en 2006 eran presentadas por colombianos. En 2008 su número cayó en picada: de 2.497 en 2007 pasó a 752 . Para 2009, eran sólo $247(8,2 \%)^{19}$. Los estudios de la Comisión Española de Ayuda al Refugiado (CEAR) mencionados nos dan luces para entender ese declive: esa disminución no se debe a que la situación colombiana esté mejorando, sino a que las peticiones están siendo sistemáticamente inadmitidas por el Gobierno español. Simplemente, los perseguidos del mundo (no sólo los colombianos) han tenido que buscar protección internacional en otros países. Es importante resaltar que en estas estadísticas no estamos incluyendo la cifra oscura, es decir, el gran número de víctimas de la violencia que debido a las trabas migratorias buscan otros medios legales o no tan legales para entrar a la Península, como son las visas de estudio, de turismo, la compra de pasaportes o la entrada irregular. De ellas encontramos varias en el trabajo de campo cualitativo. Así mismo, tampoco estamos haciendo alusión a esa sensación de inseguridad que viven un gran porcentaje de colombianos, y que se denota con mayor fuerza en las razones que se alegan para quedarse en la Ibérica (la seguridad, el poder salir a la calle a cualquier hora, etc.).

¿Quiénes están asumiendo en mayor medida los estragos de esa violencia? Las mujeres. Una prueba de lo anterior es el número de féminas desplazadas por la violencia en Colombia. A noviembre de 2009, los organismos gubernamentales habían registrado 3,2 millones de desplazados en Colombia; de ellos, el 83\% eran mujeres, niñas y niños ${ }^{20}$. Igualmente, según la Comisión de Seguimiento a la Política pública sobre el desplazamiento forzado, en el año 2008, el 52,4\% de la población desplazada interna era femenina, el 43,8\% de los hogares de esa población tenía como cabeza de familia una mujer y un gran número de esas mujeres ejercía sola dicha jefatura $(66,8 \%)^{21}$. En el mismo sentido, las entrevistas muestran que, al ser los hombres los que más pierden la vida dentro del conflicto, los que no vuelven a sus hogares y los más proclives a caer en manos de la delincuencia común, el desempleo o a ejercer la violencia en el ámbito privado, ellas se han convertido en el bastión económico, familiar y social de su entorno, siendo la migración una de las estrategias utilizadas en ello. Entre las participantes encontramos mujeres que han visto morir a su compañero sentimental o a sus hijos, o que tienen miedo de qué pueda pasar con sus vástagos si continúan en el lugar de origen, entre muchos otros casos. Parafraseando a María Fernanda, una de las entrevistadas consciente del fenómeno: "Ellas no mueren en el conflicto pero son las que padecen en vida sus consecuencias. Ellas son las que ven morir, las que sobreviven y las que reconstruyen sobre las ruinas... son las que sacan adelante la familia y los hijos...”.

Conectado con lo anterior, las féminas a través de la migración también están escapando de desigualdades y violencias de género propiamente dichas: mujeres que no encuentran cómo desarrollarse profesionalmente, mujeres cabeza de familia o

19 CEAR, La situación de las personas refugiadas en España. Informe 2009, Madrid, 2009; CEAR, La situación de las personas refugiadas en España. Informe 2010, Madrid, 2010.

20 ACNUR, Violencia de género y mujeres desplazadas. Año 2009, Nueva York, 2009.

21 GARAY, J., et. al., Séptimo Informe de verificación sobre el cumplimiento de los derechos de la población en situación de desplazamiento, Bogotá, 2008. 
mujeres maltratadas que huyen de la violencia doméstica pueblan las autopistas de la migración internacional. Un gran número de entrevistadas huían de la falta de oportunidades económicas que las estructuras de género les imponían en Colombia, ya que no encontraban trabajo o, si lo encontraban, no estaba acorde con su educación, era en zonas alejadas o con salarios más bajos. Ellas solamente están poniendo de presente el gran papel que la emigración femenina con alta formación está jugando en el fenómeno migratorio ${ }^{22}$. Así mismo, un buen porcentaje de ellas huía de parejas que las maltrataban física o psicológicamente, de estructuras familiares que les imponían un rol de feminidad específico y que, de cierta forma, las mantenían presas en sus barrios o ciudades de origen. Salir de Colombia permitía buscar nuevos horizontes o poner tierra de por medio ante una situación de violencia familiar que no se podía manejar.

Un último ejemplo. Las estadísticas muestran que el 51, 9\% de los colombianos en España y el 39\% de las mujeres migrantes de esa nacionalidad en ese país tenían pareja estable a 2003. Sin embargo, independientemente de su estado civil, el 55\% de ellos y el $60 \%$ de ellas tenían hijos ${ }^{23}$. La disparidad en las cifras en el caso de las féminas colombianas nos permite afirmar que existe un amplio número de ellas que son cabeza de familia y que migraron para contar con recursos que les permitieran hacer frente a tamaña responsabilidad.

Como ya dijimos, todas esas motivaciones que se presentan en la migración femenina hacia España no se dan en estado puro, sino que aparecen mezcladas. Por eso, entendiendo que no es una y que además son interconectadas, no se nos debe hacer raro encontrar personas como doña Rubiela, una madre vallecaucana que viaja para sacar a sus retoños adelante, mientras huye del maltrato físico y psicológico del padre de los niños e intenta evitar que sus muchachos entren en las dinámicas del conflicto:

(...) lo veía.... pero yo estaba enamorada. No lo veía. Lo veía y no lo veía. Me decía: "noo, ya cuando nos casemos, dejo de beber y tal", y yo me lo creí. Nos casamos, seguía bebiendo. Luego nace el niño, uff, en la semana bebía, el fin de semana, todos los días... de la emoción de tener un niño (...) Y... y ya empezaron los problemas ahí, ya empezaron los problemas. (...) Entonces, bueno, llegaba y me daba duro. Y yo no hacía ni ruido para que mi mamá no se enterara. Lloraba y me aguantaba los pontocones. Yo no sé cómo le aguanté tanto. Luego, quedé en embarazo de Diana, ya... ya nace Diana y él seguía igual; aun cuando había cambiado un poquito, pero no mucho. Mm. Además, ni trabajaba (...) entonces, un día le dije: "Mamá, yo me voy dejando todo. Yo voy a dejar esta relación. Ya no, ya no aguanto más. ¿Para qué? ¿Para qué mamá??'.

(...) En conclusión, que yo me harté de la situación y ya dije: "yo me voy, yo me voy". Bueno. Y Richard era muy inteligente, ocupaba el primer lugar en el colegio. Diana también era inteligente, pero Richard la superaba. Y de ver que estaba trabajando yo y que no alcanzaba ni para darle el autobús, tenía que pegarse unas caminadas como de aquí a... como de aquí más o menos qué... a

22 RAMÍREZ, C., et. al., Perfil migratorio de Colombia, OIM Colombia, Bogotá, 2010.

23 APARICIO, R., y GIMÉNEZ, C., Migración colombiana en España, Ginebra, 2003. 
la Plaza España, por decirte algo... a mí me daba una pena del niño, con lo inteligente que era; yo iba allá a las reuniones y salía como un pavo real; me decían que participaba en clase, que era el mejor, que tenían que a veces callarlo para que dejara participar a otros, bueno. Bueno, bueno, bueno. Entonces, yo decía: "cómo no voy a aprovechar esta potencia de este niño". (...).

Y como la misma idea de no.... Porque nosotros fuimos muy pobres, muy pobres, vivíamos en un barrio muy pobre donde había mucha cosa rara por ahí, mucha... y el miedo, miedo era que éstos se metieran en estos rollos. Más que todo el Richard. Y esa también fue otra de las... de los fines por los que me los traje... que no fuera a caer por ahí en... porque nosotros vivíamos en un sector.... este sector era de gente buena, pero dos, tres casas más abajo, era gente.... de todo... matones, ladrones, de todo. Sí... Entonces, ese era el miedo que... que uno no quiere que ellos se metan en esos rollos, en esas historias; siempre qué, buscando lo mejor para ellos.

Las diferentes razones que hemos expuesto, y que se ven condensadas en el relato de doña Rubiela, nos pone de presente que en los estudios sobre migración femenina debe tenerse en cuenta que el migrar, en muchos casos, es una estrategia de escape a unas injusticias de género donde se imbrican desigualdades económicas y sociales, violencia política, desarrollo profesional, etc. Al estudiar el tema, hay que preguntarse cómo hacer para que el llamado derecho a migrar se convierta efectivamente en eso, un derecho, y no en un último recurso de unas personas a desigualdades de todo tipo.

En cuanto al segundo punto, es decir, los imaginarios alrededor de las relaciones entre hombre y mujer como factor para construir la diferencia entre colombianos y españoles, en las entrevistas es posible ver cómo el colombiano relaciona el modo de ser español con irrespeto, frialdad, maltrato, descuido y falta de compromiso con la relación. ¿De dónde sacan esas conclusiones? Simple: de la no existencia de términos cariñosos -que, según Manuel, uno de los participantes, en España son tonta o retrasado-; del tono fuerte -que tanto choca a Eliana, porque pareciera que todo se arreglara a los gritos-; del pago a la americana -que para Pilar es pura tacañería-, o de la laxitud -que, en palabras de Milena, permite que la mujer coja por un lado y el hombre por el otro incluso cuando ya están casados-. Lo opuesto a esa imagen es la descripción de los vínculos amorosos entre colombianos, plagados de palabras bonitas y expresiones de afecto públicas.

Si nos fijamos, lo que tenemos a la vista son estructuras de clase y género y concepciones de masculinidad y feminidad con roles específicos que, hasta cierto punto, se legitiman a través de estereotipar al otro e incluso deformarlo. Por una parte, tenemos la imagen de unos hombres españoles que, o ejercen su poder a través de la fuerza (como se puede concluir de las recurrentes noticias sobre violencia doméstica), o han perdido toda su autoridad y “su mujer los maneja”. Por otro, la de unas féminas ibéricas cuya independencia no se percibe como una liberación, sino como una feminismo extremo. En palabras de Mariana, colombiana casada con un hombre de su misma nacionalidad: 
"Yo veo que hay... hay los dos extremos, que a mí... que yo considero que son muy malos: hay mucho machismo y mucho feminismo. Demasiado, exagerado. Porque en la pareja que predomina el machismo, suele ser el hombre que maltrata, que pega, que amenaza, que tal, que lo vemos todos los días, ¿no?, los números de muertas que van. Y en el hogar que no es así, entonces, es la mujer que domina, que gobierna, que... que anula casi por completo al hombre, que el hombre no tiene ni voz ni voto en esa casa. A mí me parece muy nocivo tanto lo uno como lo otro para una pareja".

Así mismo, podemos encontrar que la comparación se utiliza para idealizar lo propio. Escuchemos el paralelo que realiza Eliana, una caleña que cuida muy bien de su marido:

(...) las mujeres son muy independientes. Lo sé porque me ha tocado vivirlo en casas, ¿no?, donde ellas van y se toman un café y se quedan horas, horas, pasan horas enteras en una cafetería; mientras que nosotras no somos de esas costumbres. Nosotras, las mujeres colombianas, ehh, si salimos, claro, se lo comunicamos a nuestra pareja, pero siempre estamos pendientes a llegar a la hora para servirles la comida, que aquí es cena. Pero yo como mujer colombiana, yo no me pierdo tanto tiempo con mis amigas y "ala, ahí tú te quedas", como dicen, ¡no! No, no, porque yo vengo de un hogar donde mi madre se dedicó mucho a mi padre, ¿sí?, lo atendía muy bien y siempre estaba ahí, ella le servía la comida y todo eso. Entonces, yo también guardo como esa, esa, ese... esa, esa, esa similitud. Acá veo la gente muy independiente, que el marido llega y... pues se sirve él mismo; o si no hay nada, se llegan ellos a hacer la cena. No, no, no... nosotros no. O sea, la costumbre mía es siempre dejar la comida hecha; si tengo que salir, aviso, pero... si salgo con las amigas, pues no trato de tardarme mucho, sino dentro de la normalidad... Eso lo veo, la mujer es muy independiente, la mujer es muy independiente; no está tan pendiente de su marido como la mujer colombiana. La española es muy independiente en ese sentido. Creo que tienen un trato bastante fuerte para con sus maridos, un tono de voz muy reacio, muy alto; cosa que en la mujer suramericana, y especialmente en la colombiana, no se ve. El trato es más cálido... ehh... somos muy cariñosas, muy sutiles; mientras que la mujer española no tiene tanto ese carisma de dar, de ese romanticismo, todo esto... jsomos muy distintas! (enfatiza). Las unas de las otras somos bastante distintas, sí, sí, sí.

Una de las consecuencias de esta autopercepción idealizada de las relaciones entre parejas heterosexuales es el mantenimiento de ciertas desigualdades de género que, inclusive, y a pesar de la contradicción que esto pueda generar, son utilizadas como mecanismos de resistencia. Por una parte, justifica la identificación de feminidad con sumisión (las mujeres colombianas son más femeninas porque son más dulces y sumisas), lo que permite retroalimentar una construcción de lo femenino a la postre compartida por las sociedades colombiana y española y que se ve reflejada en que los hombres mayores busquen casarse con mujeres latinoamericanas. El 66\% de las 
uniones de las colombianas en el año 2005 fue con ciudadanos de la Península ${ }^{24}$; estadísticas locales y la investigación cualitativa permiten afirmar que un porcentaje relevante estaba constituido por uniones entre mujeres cabeza de familia y hombres mayores que ellas. Por otra, la feminidad es un activo que cotiza a las mujeres en el mercado marital español; es como si ellas tuvieran un bien que puede ser utilizado como una de las armas de la lucha por la supervivencia que las obligó a migrar. Casarse con un español permite conseguir papeles, mayor estabilidad económica y facilidad de reagrupar a la descendencia. De hecho, según algunos autores, lo es ${ }^{25} \mathrm{y}$, en algunos casos, el trabajo cualitativo lo demuestra.

Una segunda consecuencia de esta percepción maquillada de lo propio es la invisibilización de la violencia de la que son víctimas las mujeres colombianas cuando se contrasta con la gran notoriedad que adquiere la violencia intrafamiliar en España. Los españoles son unos violentos en relación con los colombianos. Aquí encontramos ejemplificado una variante del que Sarah Song ${ }^{26}$ denomina "efecto distractor”, con el cual pretende mostrar cómo el rechazo a las normas y prácticas de un grupo cultural sirve para desviar la atención de los aspectos censurables de la propia cultura. En el caso de los colombianos, el rechazo a la intimidación que ejercen los hombres españoles hacia sus mujeres desvía la atención de las violencias que también existen en las relaciones entre colombianos. Muchos de los entrevistados de la investigación han olvidado que eso que tanto reprochan a los ibéricos también sucede en la colonia colombiana y en su lugar de origen.

Tal efecto distractor también se pone de manifiesto en la manera en que la sociedad colombiana construye sus relaciones con los pueblos indígenas, con esos “otros” que conviven al interior de nuestras fronteras; en particular, en la manera en que concilia las demandas de equidad de género y respeto a la diversidad cultural. A ello se refiere el segundo de los casos que presentaremos, el cual nos servirá para ejemplificar la "feminización de la supervivencia" que tiene lugar en contextos multiculturales.

\section{EL CUBRIMIENTO POR LOS MEDIOS DE COMUNICACIÓN DE LA SANCIÓN IMPUESTA A LAS INDÍGENAS EMBERA DEL CHOCÓ QUE VENDEN SU CABELLO}

Hace cerca de dos años el programa Séptimo Día, que se transmite por el Canal Caracol en Colombia, presentó un reportaje titulado "El cabello sagrado de la belleza”, en el que se ocupa del caso de las indígenas Embera del Chocó que son sancionadas al interior de su comunidad por cortarse el cabello para venderlo en las

24 GARCÍA DOMÍNGUEZ, M., Migración colombiana del AMCO hacia España, Santo Domingo, 2007; MTAS, Anuario Estadístico de Extranjería 2006, España, 2006.

25 Ver, por ejemplo, GARCÍA DOMÍNGUEZ, M., Ibídem.

26 SONG, S., Majority Norms, Multiculturalism, and Gender Equality, en The American Political Science Review, Nov 2005, 99, 4, pp. 473 - 489. 
peluquerías de Quibdó, donde sus largas, lisas y sanas cabelleras son utilizadas para hacer extensiones capilares para las mujeres afrodescendientes de la zona ${ }^{27}$.

Conviene aclarar que el objeto de nuestro análisis no será directamente el conflicto que origina entre los Embera del Resguardo La 21 en Chocó el que algunas mujeres de la comunidad corten su cabello para venderlo. No porque el interés del caso no lo amerite, sino porque hacerlo con rigor requeriría una aproximación etnográfica que aún no hemos realizado. En su lugar, nos proponemos examinar la relación entre injusticias culturales, socioeconómicas y de género a partir del modo en que este medio de comunicación se ocupó del tema.

En el reportaje del programa Séptimo Día se identifican (al menos) tres situaciones problemáticas:

En primer lugar, la construcción de un patrón de belleza para la mujer negra que lleva a desvalorizar características propias, para adecuarse a un canon de belleza que les impone adquirir en el mercado un "bien” que ellas no poseen (cabello lacio). Concentrarnos en esta arista del problema nos llevaría a ocuparnos de cuestiones como la devaluación de ciertos rasgos físicos de la población afrodescendiente y, ligada a ésta, la desigualdad que se instaura entre las mujeres que pueden comprar una cabellera lacia y quienes no pueden hacerlo. Pero no será esta la cuestión en la que nos concentremos.

En segundo lugar, algunas mujeres indígenas que poseen este "bien” concurren al mercado para ofertarlo. Sin embargo, esta oferta no es el resultado de la libre concurrencia, sino de la necesidad de proveer recursos para asegurar la subsistencia propia de la de las personas a su cargo. Esa imagen de las comunidades indígenas, como entidades autárquicas sin contacto alguno con el resto de la sociedad, no se compadece con una realidad en la que los indígenas, con muy pocas excepciones, están inmersos en relaciones de intercambio, que muchas veces discurren a través del mercado. La mayoría de los bienes con los que las mujeres indígenas suelen concurrir al mercado (productos agrícolas, artesanías, venta de mano de obra para el servicio doméstico, entre otras) no garantizan por sí solos la adquisición de bienes básicos para la subsistencia ni son tan bien valorados como una negra, larga y lacia cabellera.

En tercer lugar, la sanción que las autoridades indígenas imponen a las mujeres de su comunidad que concurren a ofertar este "bien” en el mercado. La cabellera larga tiene en la comunidad un significado asociado a la virtud femenina. Cortarse el cabello está asociado a la pérdida de la virtud, a la prostitución, según lo explica ante las cámaras de televisión el gobernador del Resguardo La 21.

El reportaje se concentra en cuestionar la injusticia derivada de la imposición de la pena de cepo a las mujeres emberas que venden su cabello y, por esta vía, la identificación del cabello largo de las mujeres como un símbolo de virtud femenina. Esta lectura del fenómeno parecería entonces plantear un conflicto entre el derecho

27 El reportaje puede verse en: http://www.caracoltv.com/producciones/informativos/ septimodia/video153892-el-pelo-sagrado-de-la-belleza. Última revisión, Julio 1/2011. 
de las mujeres embera a disponer de su propio cuerpo, a llevar su cabello como les plazca y, por otro lado, el reconocimiento del derecho de la comunidad a sancionar un comportamiento que se juzga reprochable, en ejercicio del derecho colectivo a la autonomía jurisdiccional.

De acuerdo con esta lectura, este sería un perfecto ejemplo de la llamada "paradoja de la vulnerabilidad multicultural”, esto es, la muestra de cómo los costos de una política de reconocimiento de la diversidad cultural pueden llegar a estar inequitativamente repartidos y a gravar excesivamente, acentuando la situación de vulnerabilidad de algunos sectores de población, en este caso las mujeres indígenas. La literatura feminista ha destacado el hecho de que el multiculturalismo puede tener el efecto de reforzar la inequidad de género dentro de los grupos minoritarios, pues para garantizar el derecho a la reproducción cultural de estos grupos, se autoriza que, a través de sus normas y prácticas, algunos de los miembros del grupo opriman a los sectores más vulnerables del grupo o "minorías internas"28.

Aun cuando el reportaje no lo hace explícito, una conclusión fácil que podría extraer aquél espectador que haga una valoración superficial de la situación, es la necesidad de establecer límites a la autonomía jurisdiccional de las comunidades indígenas, de modo tal que se impida la sanción de las mujeres que acuden al mercado a vender su cabello.

Esta manera de abordar la situación tiene el efecto de reforzar prejuicios “alterofóbicos”, al mostrar las bárbaras costumbres de los indígenas, y constituye una muestra de lo que Sarah Song denomina el "efecto distractor”, para destacar el hecho de que concentrarse en el examen crítico de las normas y prácticas de las culturas minoritarias puede contribuir a desviar la atención o bien a legitimar las normas y prácticas de la cultura mayoritaria. Con esto no se quiere sugerir que debamos mirar hacia otro lado y no prestar atención a situaciones problemáticas como las que plantea el reportaje de Séptimo Día, pues esta renuncia a considerar al otro como interlocutor en el debate moral que suscitan estas situaciones de inequidad de género, simplemente pretextando el respeto a su diferencia cultural, supondría una forma del "falso reconocimiento" del otro. La propuesta de Song, que retomo para el análisis, es que nuestras normas y prácticas deben ser analizadas a la vez que se hace lo propio con las de otras culturas, para así evitar pasar por alto que estos efectos desviantes pueden reforzar jerarquías de género entre culturas y alimentar discursos de superioridad racial y cultural dentro de la cultura dominante $^{29}$.

Atendiendo a la recomendación que nos formula Sarah Song, quisiéramos llamar la atención sobre dos cuestiones que el reportaje no problematiza, sino que, por el contrario, naturaliza, los asume como parte del paisaje en el que transcurre la

28 SHACHAR, A., "The Paradox of Multicultural Vulnerability”, en JOPPKE, C., y LUKES, S. (comps.), Multicultural Questions, Oxford, Oxford University Press, 1999, pp. 87-111; SACHAR, A., "On Citizenship and Multicultural Vulnerability”, Political Theory, Vol. 28, No. 1, 2000, pp. 64-89.

29

SONG, S., op. cit., p. 486. 
historia narrada, pero que constituyen dos situaciones de injusticia profundamente arraigadas en nuestra sociedad:

En primer lugar, la valoración que hacen los hombres embera del cabello largo de las mujeres como símbolo de su virtud no constituye una seña de "diferencia cultural radical” respecto de la sociedad no indígena, sino que, por el contrario, pone de manifiesto un elemento común a ambas sociedades (y a muchas otras): el control sobre el cuerpo femenino, concebido como un territorio en el que se inscriben trazas de esa dominación patriarcal a la que antes nos referíamos.

Dejando de lado cuestiones tan embarazosas como el control de la natalidad -que se manifiesta en algunas comunidades indígenas con la prohibición del uso de anticonceptivos y, en un entorno que nos es más cercano, con la cruzada emprendida por el Procurador Alejandro Ordoñez y los sectores conservadores de la ciudad de Medellín en contra del proyecto de Clínica de la Mujer- para concentrarnos en un asunto en apariencia más trivial, también nuestra sociedad percibe el cabello largo como un símbolo de feminidad. Incluso existe en nuestro medio una sanción social difusa en contra de la mujer que se atreva a llevar el cabello corto, y aunque su carácter aflictivo no se equipare al de una pena corporal como el cepo, en cualquier caso no deja de manifestarse con mayor o menor intensidad, según el sector social al que se pertenezca y la dependencia o autonomía de la que goce una mujer. Ejemplo de esto fue la controversia que generó la decisión de la Señorita Colombia 2009, Michelle Rouillard, de cortarse el cabello meses antes de asistir al certamen de Miss Universo.

Pero además en la literatura podemos encontrar numerosos ejemplos de esta identificación del cabello largo con la presencia inquietante de la naturaleza, en contraste con el cabello corto como signo de ingreso en la civilización. Paralela a ésta corre la identificación entre el cabello largo como símbolo de feminidad y del cabello corto como insignia de virilidad.

Michelle Perrot, en su trabajo titulado "Mi historia de las mujeres”, nos recuerda que apóstol Pablo escribe a los Corintios: "La naturaleza misma nos enseña que para un hombre es deshonroso tener el cabello largo, pero que una mujer se glorifica por su larga cabellera. Le ha sido dado el cabello en lugar del velo" ${ }^{30}$. Velo que, como es conocido, representa en la tradición judeo cristiana y en la musulmana un símbolo de virtud y de pudor.

También cita a Soren Kierkegaard, quien en su Diario de un seductor, escribe:

¿Qué hay más bello que la abundante cabellera de una mujer, que esa profusión de bucles? Sin embargo, es un signo de su imperfección, según las Escrituras, que ofrecen diversas razones para ello ¿Y no es éste el caso? Mira a la mujer inclinándose casi hasta tocar el suelo con sus pesadas trenzas, parecidas a sarmientos floridos que la unen a la tierra; ¿no es ella una naturaleza más imperfecta que el hombre, que tiene la mirada vuelta al cielo y

30 PERROT, M., op. cit., p. 66. 
que simplemente toca el suelo? Y sin embargo, esa cabellera es su belleza; más aún, su fuerza. Pues por ella...cautiva al hombre, lo encadena y lo ata a la tierra" 31 .

Por eso, más eficaz y más radical que alimentar la indignación o el extrañamiento frente a esos otros que castigan a sus mujeres, como prostitutas, porque venden su cabello, nuestra sociedad tendría que mirarse en ese espejo para reconocer también aquí muchas de esas prácticas que impiden a las mujeres decidir de manera autónoma sobre su cuerpo y, en su lugar, lo siguen concibiendo como un territorio sobre el que lo masculino tiene plena jurisdicción. Promover, a través de la educación, de los medios de comunicación, esos cambios en las diversas formas de violencia social que se ejerce frente a la mujer, entre ellas la imposición de un cierto modelo de feminidad o de virtud, repercutirá positivamente en el respeto de la autonomía de todas las mujeres, también de las indígenas, para tomar decisiones relativas a su cuerpo. Ya los propios emberas se encargarán de revisar la decisión de castigar con cepo dicha práctica, como se han encargado ellos mismos de revisar la de la ablación de clítoris, o los U’wa revisaron la práctica del abandono de gemelos.

La segunda injusticia sobre la que queremos llamar la atención a propósito del reportaje de Séptimo Día es la que obliga a algunas personas, en este caso a las mujeres emberas, a vender partes de su cuerpo. Porque la decisión de cortar sus cabellos no obedece al capricho de cambiar de imagen, ni a una afirmación de independencia frente a esa marca de feminidad que representa el cabello largo, sino a la necesidad de entregar en el mercado un "bien" que ellas poseen y del que necesitan despojarse para asegurar su sustento y el de sus hijos.

La decisión de desafiar las normas de la comunidad (o en el caso de las mujeres que tengan introyectado el patrón de valoración del cabello femenino, de despojarse de un símbolo de virtud) para obtener doscientos mil pesos por su cabellera, no es comparable a la decisión de la Señorita Colombia de desafiar los cánones estéticos dominantes al despojarse de su larga y cuidada cabellera. En el primer caso no puede hablarse de una decisión realmente libre, sino de una actuación motivada por un verdadero estado de necesidad. En el reportaje esta cuestión no es problematizada en absoluto. De hecho, en algún pasaje, luego de mostrar la satisfacción de la mujer negra con sus extensiones capilares recién puestas y la de la mujer indígena al salir de la peluquería con el dinero en la mano, se dice que "hasta aquí la historia tendría un final feliz". Vale la pena entonces preguntarse ¿hasta qué punto hemos naturalizado las injusticias de redistribución y hemos convertido este tipo de situaciones en parte del aire social que respiramos, un dato respecto del cuál nada podemos hacer?

Coincidimos con Nancy Fraser cuando afirma que la lucha por el reconocimiento de la diferencia no puede desligarse de la lucha por la redistribución equitativa del acceso a los bienes básicos, e incluso consideramos que esta última exigencia de justicia adquiere prioridad sobre la primera, especialmente en contextos de flagrante inequidad social como el que se presenta en Colombia. En tales circunstancias cabe

31 Ibidem, p. 70. 
afirmar que no puede darse un avance real en términos de reconocimiento de las diferencias si estos no se acompañan de una política orientada a corregir las graves injusticias de redistribución, pues, como señala Fraser, "las diferencias culturales sólo pueden elaborarse libremente y ser mediadas democráticamente sobre la previa garantía de la igualdad social”32.

\section{CONCLUSIONES}

El análisis de algunas de las situaciones que viven los migrantes colombianos en España y las mujeres indígenas embera en el Pacífico colombiano nos proporciona unas cuantas reflexiones a tener en cuenta en investigaciones a futuro en este tipo de temas.

En primer lugar, los dos casos examinados nos permiten apreciar que las desigualdades de género, derivadas de relaciones patriarcales de dominación, mezclan a su vez injusticias de reconocimiento y de redistribución inequitativa del acceso a oportunidades y recursos económicos. Lo vimos en la importancia estadística y las motivaciones que impulsan a las mujeres colombianas a migrar a España. También lo percibimos en el caso de las mujeres embera que se ven obligadas a vender su cabello para conseguir el sustento de sus familias. Eso significa que la superación de las desigualdades de género requiere adoptar medidas que, de acuerdo con una concepción "bivalente” de la justicia como la que propone Nancy Fraser ${ }^{33}$ :

- Distribuyan los recursos materiales de forma tal que aseguren la independencia y la voz de los miembros de la sociedad, sin obligar a que algunos de ellos tengan que migrar o que vender partes de su cuerpo para garantizar su supervivencia.

- Excluyan aquellos patrones culturales que menosprecian sistemáticamente algunas categorías de personas y las cualidades asociadas con ellas. Esto significa corregir aquellos patrones culturales que devalúan lo femenino frente a lo masculino o que asocian la feminidad con determinados rasgos como la sumisión o el cabello largo, y que terminan por imponerse a las mujeres, en lugar de ser el resultado de elecciones autónomas.

En segundo lugar, también podemos concluir con Sarah Song que, si reconocemos que las culturas no son totalidades separadas, claramente distinguibles y coherentes a su interior, sino que son internamente conflictivas y se encuentran en permanente interacción y mutua constitución con otras culturas, entonces, el problema para el feminismo no es tanto "multiculturalismo vs equidad de género", sino más bien "patriarcado vs equidad de género"34. Formular el problema en estos términos

32 FRASER, N., op. cit., p. 242.

33 FRASER, N. "La justicia social en la época de la política de la identidad: redistribución, reconocimiento y participación”, en Justicia Social. Estudios Ocasionales. Cátedra Ciro Angarita, Bogotá, Universidad de Los Andes, 2007, pp. 9-29, aquí p. 24.

34

SONG, S., op. cit. 
permite la consideración del rol que las normas e instituciones de las culturas, sean éstas mayoritarias o minoritarias, juegan en la formación o reforzamiento de las prácticas de las otras culturas. Pero también permite el examen de factores no vinculados a la diferencia cultural, tales como las injusticias socioeconómicas, que obstaculizan las luchas por la equidad de género. 